ZIBELINE INTERNATIONAL

ISSN: 2521-0874 (Print)

ISSN: 2521-0505 (online)

CODEN : AIMCCO

\title{
ANALYSIS OF PUBLIC DATA ON SOCIAL NETWORKS WITH IBM WATSON
}

Zoltan Balogh

Computer Science Department, Corvinus University of Budapest

Halal Product Research Institute, Universiti Putra Malaysia.

Gorresponding Author Email: zoltan.balogh2@uni-corvinus.hu

This is an open access article distributed under the Creative Commons Attribution License, which permits unrestricted use, distribution, and reproduction in any medium, provided the original work is properly cited.

\section{ARTICLE DETAILS}

Article History:

Received 12 December 2017

Accepted 10 January 2018

Available online 19 February 2018

\section{ABSTRACT}

As more and more people are using the the social networks, they leave enormous amount of digital footprints of their personality available to them with every click. In this exploratory research, the publicly available data is collected from the biggest social network (Facebook) and sent to IBM Watson to reveal hidden personality traits that the posts and the profile pictures might contain. Watson is the name of the the cognitive computing system developed by IBM. It has several subsystems including the Personality Insights, that extracts personality traits from text and the Visual Recognition, that can be taught to analyze pictures. Firstly, the posts of Facebook users would be sent to Watson Personality Insights to get their Big5 personality traits, then their profile images and their personality traits will be linked to train the Visual Recognition. With a high number of input, the API probably could output the personality traits with high significance.

\section{KEYWORDS}

IBM Watson, personality insights, visual recognition, social network, neural network.

\section{INTRODUCTION}

Neural networks can be trained to recognize the contents of an image or to identify specific details of it. If the neural network is trained on a quality input, then it might be better at image recognition than the human beings. Subsystems of the IBM Watson used in this research are using neural networks.

\section{LITERATURE REVIEW}

According to a study of Leslie A. Zebrowitz and Mary Ann Collins there exists a link between the facial appearance and the inner characteristics of the subject. Moreover, the psychological state, and the demographic traits are also detectable. It depends on the observer whether he can detect and interpret these signs. The existence of such links between facial appearance and character is supported by the fact that people can accurately judge others' character, psychological states, and demographic traits from their faces [1].

The human brain needs as little as $100 \mathrm{~ms}$ exposure to detect some of the personality traits of unfamiliar faces [2]. Deep neural networks outperform the human beings in the detection of sexual orientation of people by analyzing their faces. A recent study proved that deep neural networks can be trained with a sample of 35.000 instances to identify the sexual orientation of the person depicted on the image with a confidence level of $91 \%$ [3].

\section{RESEARCH}

This research is still ongoing. As there are successful researches that personality traits can be deducted from the traces of visitors left on social networks. This is an exploratory research that uses the public post messages and profile picture of a user and tries to identify the most common personality traits and aspects, including Big5, IQ score, satisfaction with life, religious and political views. In psychology, the Big5 personality traits are attributes that can describe human personality. These five factors are openness, conscientiousness, extraversion, agreeableness, and neuroticism.

In 2012, the researchers at The Psychometrics Centre at the University of Cambridge analyzed a dataset of more than 58,000 volunteers who provided access to their Facebook likes and filled out several psychological tests. Then they used the collected data to develop a public API that can predict private properties of individuals from their Facebook Likes [4].

\section{METHODOLOGY}

In 2012 April, a tracker script was embedded into the e-learning system of the Corvinus University of Budapest, that saved all the posts created by the visitor with their prior consent to database. The script ran for 2 weeks and 139 visitors allowed the saving of the personal data stored on Facebook.

The input data for the research comes from two different sources: the Facebook data of the visitors of Corvinus University of Hungary and the data received from my Personality Project. The text data will be fed to IBM Watson's Personality Insights API and get the Big5 traits of the visitors. Only the visitors that have the minimum of 7000 characters of total posts can be included to output a strong result. Then the profile pictures linked with personality traits will be fed to IBM Watson's Visual Recognition API. The API will be trained to detect the personal traits of the person that the picture depicts and might be invisible for the human eye.

\section{EXPECTED OUTPUT}

Using neural networks to detect personality traits from texts and photos is still in its early stage. There is a huge potential in this technology as better targeted advertisements can be sent to visitors. If the personal characteristics of the visitor is known, they can be offered products that might better fit their needs. The goal of this ongoing research is to find out whether it is possible to detect personality traits with the neural network implemented in IBM Watson.

\section{REFERENCES}

[1] Zebrowitz, L.A., Collins, M.A. 1997. Accurate Social Perception at Zero Acquaintance: The Affordances of a Gibsonian Approach. Personality and Social Psychology Review, 204-223.

[2] Todorov, A., Said, C.P., Engell, A.D., Oosterhof, N.N. 2008. 
Understanding evaluation of face on social dimensions. Trends in Cognitive Sciences, 12 (12), 455-460.

[3] Kosinski, M., Wang, Y. 2017. Deep neural networks are more accurate than humans at detecting sexual orientation from facial images. 7 september. [Online]. Available: https://www.gsb.stanford.edu/faculty- research/publications/deep-neural- networks-are-more-accurate-humansdetecting-sexual.

[4] Kosinski, M., Stillwell, D., Graepel, T. 2013. Private traits and attributes are predictable from digital records of human behavior. PNAS, 5802-5805. 